Review

\title{
FUT8 and Protein Core Fucosylation in Tumours: From Diagnosis to Treatment
}

\author{
Chengcheng Liao' ${ }^{1}$ Jiaxing An³, Suqin $\mathrm{Yi}^{3}$, Zhangxue Tan${ }^{1}$, Hui Wang 3 , Hao Li' ${ }^{1}$, Xiaoyan Guan ${ }^{4}$, Jianguo \\ Liu1, Qian Wang ${ }^{1,2}$ \\ 1. Special Key Laboratory of Oral Disease Research, Higher Education Institution in Guizhou Province, School of Stomatology, Zunyi Medical University, \\ Zunyi 563006, China. \\ 2. Microbial Resources and Drug Development Key Laboratory of Guizhou Tertiary Institution, Life Sciences Institute, Zunyi Medical University, Zunyi \\ 563006, China. \\ 3. Department of Gastroenterology, Affiliated Hospital of Zunyi Medical University, Zunyi 563000, China. \\ 4. Department of Orthodontics II, Hospital of Stomatology, Zunyi Medical University, Zunyi 563000, China. \\ $\triangle$ Corresponding author: Qian Wang, E-mail: qianwang07@126.com
}

(c) The author(s). This is an open access article distributed under the terms of the Creative Commons Attribution License (https://creativecommons.org/licenses/by/4.0/). See http://ivyspring.com/terms for full terms and conditions.

Received: 2021.01.16; Accepted: 2021.04.27; Published: 2021.05.13

\begin{abstract}
Glycosylation changes are key molecular events in tumorigenesis, progression and glycosyltransferases play a vital role in the this process. FUT8 belongs to the fucosyltransferase family and is the key enzyme involved in $\mathrm{N}$-glycan core fucosylation. FUT8 and/or core fucosylated proteins are frequently upregulated in liver, lung, colorectal, pancreas, prostate,breast, oral cavity, oesophagus, and thyroid tumours, diffuse large B-cell lymphoma, ependymoma, medulloblastoma and glioblastoma multiforme and downregulated in gastric cancer. They can be used as markers of cancer diagnosis, occurrence, progression and prognosis. Core fucosylated EGFR, TGFBR, E-cadherin, PDI/PD-LI and $\alpha 3 \beta 1$ integrin are potential targets for tumour therapy. In addition, IGgl antibody defucosylation can improve antibody affinity, which is another aspect of FUT8 that could be applied to tumour therapy.
\end{abstract}

Key words: FUT8; core fucosylation; EGFR; TGF- $\beta$ receptor; E-cadherin; PD1/PD-L1; a3 $\beta 1$ integrin.

\section{Introduction}

Glycosylation is a ubiquitous modification that occurs on the proteins and lipids of all living cells, and these polysaccharides are essential for life. Protein glycosylation is involved in the basic molecular and cellular biological processes in the development of cancer, such as cell signalling and communication, tumour cell migration and invasion, cell matrix interaction, tumour angiogenesis, immune regulation and metastasis formation [1]. To date, more than 180 glycosyltransferase genes have been discovered to be involved in the biosynthesis of glycans [2-3]. It has become possible to manipulate glycosyltransferases to modify the structure of oligosaccharides to examine the effects of these modifications on certain events [4-5]. Fucosyltransferase (FUT) is essential in many physiological and pathological activities, such as inflammation, bacterial and viral infections, tumour metastasis, and genetic diseases [6], and it is involved in regulating the fucosylation of O-glycans and N-glycans.

To date, 13 species of fucosyltransferase have been identified and they are divided into five categories: The first category, including FUT1 and FUT2, relates to the synthesis of $\mathrm{a}-1,2$ fucosidic bonds; the second category, including FUT3, 4, 5, 6, 7 and 9 , is related to the synthesis of a-1, 3/4 fucoside bonds; the third category, mainly FUT8, is related to the synthesis of a-1, 6 fucoside bonds; and the fourth category includes FUT10 and FUT11. The enzyme activities of FUT10/11 are still under debate, but there is a paper showing the activity of these enzymes [7]. The last category includes Pofut1 and Pofut2, which modify EGF-like domains and thrombospondin repeats (TSRs), respectively [8]. Current research shows that FUT8, Pofut1 and Pofut2 are essential for the normal development of mice, indicating the 
importance of some members of FUTs in the normal physiological functions of the body [9]. FUTs are involved in tumour regulation, especially FUT8, which is considered to be directly related to tumours [9-10].

The FUT8 gene is located on chromosome 14q24.3. Its chromosome location is different from any other fucosyltransferase genes reported so far, and its structure is also quite different, suggesting that FUT8 may have unique biological significance [11]. No oligosaccharide structure with a core fucose was found in mice after FUT8 gene knockout, suggesting that FUT8 may be the only fucosyltransferase involved in core fucosylation [12]. Mammalian FUT8 is a type II transmembrane glycoprotein, which is mainly concentrated in the Golgi body [13]. It is a catalytic enzyme whose function is to transfer GDP fucose to the initial $\mathrm{N}$-acetylglucosamine (GlcNAc) residue of the $\mathrm{N}$-glycan core by forming a-1,6 glycosidic bonds, which constitutes the core fucose [14].

FUT8 consists of a catalytic domain, an $\mathrm{N}$-terminal a-helical domain and a C-terminal Src homology 3 (SH3) domain. The $\mathrm{SH} 3$ domain is usually mediates protein-protein interactions by recognizing a proline-rich peptides in cytoplasmic signal transduction molecules [15]. No other glycosyltransferases have been found to have $\mathrm{SH} 3$ domains. The SH3 domain binds to ribophorin 1 (RPN1) to strictly control the catalytic activity and positioning of FUT8, thereby promoting the activity of FUT8 and the core fucosylation [16]. FUT8 follows the $\mathrm{S}_{\mathrm{N}} 2$ mechanism and unfolds a series of loops and an a-helix, which all contribute to the formation of binding sites, and an exosite composed of one loop structure and one $\mathrm{SH} 3$ domain is responsible for recognizing branched sugars [17]. When bound to the acceptors, FUT8 requires the presence of a terminal GlcNAc moiety on the a1,3 arm of the N-glycan [18]. The process of FUT8 capturing the substrate and the formation of the salt bridge between GDP and the two flow cycles are largely driven by Arg365 [19]. In addition, Glu273 and Lys369 of FUT8 directly play a catalytic role (Glu273 acts as a catalytic base, and Lys369 transfers a proton from Glu273 to the leaving phosphate group of the GDP-fuc substrate) [19].

In this review, we describe the diagnostic value of FUT8 and glycoproteins with core fucosylation for liver, lung, colorectal, pancreas, prostate, oral cavity, oesophagus, thyroid and stomach tumours (Table 1). More importantly, many pivotal glycoproteins on human tumour cells are highly core fucosylated, and core fucosylation is essential for the functions of these proteins. We summarized the existing evidence and discussed the impact of these core fucosylated glycoproteins on tumors to further clarify the feasibility of targeting FUT8 for the treatment of tumors. In addition, applying the FUT8 knockout mammalian cell lines to antibody production can obtain antitumour monoclonal antibodies with better performance, defucosylated IGg1 antibodies have been applied in tumour therapy.

\section{FUT8 and glycoprotein core fucosylation as tumour diagnostic markers}

FUT8 is frequently upregulated in human tumours [20-21]. A recently published meta-analysis showed that FUT8 expression levels are associated with the overall survival of breast cancer, non-small-cell lung cancer, glioma, diffuse large B-cell lymphoma, and gastric cancer [21]. FUT8 is also related to the disease-free survival of breast cancer, non-small cell lung cancer and colorectal cancer, and the recurrence-free survival of pancreatic ductal adenocarcinoma [22]. Of course, the diagnostic value of FUT8 in tumours is not limited to its abnormal expression of itself, and the changes in glycoprotein core fucosylation mediated by FUT8 are also very valuable diagnostic indicators.

\subsection{AFP-L3, FUT8 and core fucosylated haptoglobin, ceruloplasmin for hepatocellular carcinoma diagnosis}

Hepatocellular carcinoma (HCC) is the most common type of liver cancer. Currently, only a-fetoprotein (AFP), core-focused AFP (AFP-L3) and des-gamma-carboxy prothrombin (DCP) are used as biomarkers for HCC [23]. AFP-L3 has a high sensitivity for early-stage HCC [24]: reflects the progression and migration of HCC, such as DCP and AFP [25]; and can be used to predict the risk of HCC in nonalcoholic fatty liver disease (NAFLD) patients [26]. More importantly, the increase in AFP-L3 can further rule out the possibility of other liver diseases, such as hepatitis and cirrhosis, thereby reducing the false-positive results of AFP in the diagnosis of HCC [27-28]. AFP-L3 has been approved by the U.S. Food and Drug Administration (FDA) as a diagnostic serum biomarker for HCC.

FUT8 participates in AFP core fucosylation [29], and can be used as a marker to assess HCC progression [30]. The expression of FUT8 promotes the proliferation and invasion of HCC [31], and mediates multidrug resistance in human liver cancer through the PI3K/Akt signalling pathway [32]. FUT8 has good correlation with AFP. A recent study showed that in HCC with low AFP levels, several sialylated rather than core-fucosylated triantennary were glycans uniquely increase, while in high AFP 
HCC, many core-fucosylated biantennary, mixed glycans and dimeric glycans were uniquely increased [33].

Haptoglobin ( $\mathrm{Hp})$ is an abundant human plasma protein. The main biological function of $\mathrm{Hp}$ is to remove the haemoglobin $(\mathrm{Hb})$ with high affinity, thereby preventing $\mathrm{Hb}$-mediated kidney and blood vessel damage, iron loss, and the oxidation of heme-based proteins and membrane lipids. Hp also regulates angiogenesis, nitric oxide homeostasis, immune response and prostaglandin synthesis [34]. An increased degree of $\mathrm{Hp}$ core fucosylation can distinguish patients with early HCC and those with liver cirrhosis and provide a potential marker for the early detection and prediction of HCC in patients with liver cirrhosis [35]. Only a small amount of core fucosylation is detected in haptoglobin in chronic disease patients [36], making core fucosylated haptoglobin a valuable HCC indicator similar to AFP-L3.

Table 1. FUT8 and core fucosylated glycoproteins as tumours diagnostic markers.

\begin{tabular}{|c|c|c|c|c|}
\hline Tumours & Diagnostic markers & Application & $\begin{array}{l}\text { Compared with } \\
\text { normal level }\end{array}$ & Reference \\
\hline \multirow{5}{*}{$\begin{array}{l}\text { Hepatocellular } \\
\text { carcinoma (HCC) }\end{array}$} & AFP-L3 & Diagnose HCC & $\uparrow$ & [24-28] \\
\hline & FUT8 & A marker to assess HCC progression & $\uparrow$ & {$[30,33]$} \\
\hline & Core fucosylated haptoglobin & Diagnose HCC & $\uparrow$ & [34-36] \\
\hline & Core fucosylated ceruloplasmin & $\begin{array}{l}\text { Distinguish between alcohol-related HCC and } \\
\text { alcohol-related cirrhosis }\end{array}$ & $\uparrow$ & {$[37,38]$} \\
\hline & Core fucosylated a-1-antitrypsin (A1AT) & Predict HCC metastasis & $\uparrow$ & [39] \\
\hline \multirow[t]{3}{*}{ Pancreatic cancer (PC) } & FUT8 & $\begin{array}{l}\text { Significantly related to lymph node metastasis and } \\
\text { recurrence-free survival of PDAC patients }\end{array}$ & $\uparrow$ & {$[21,50]$} \\
\hline & Ribonuclease 1 (RNase 1) & Serum markers of pancreatic cancer & $\uparrow$ & [53] \\
\hline & Total serum core-Fuc-Hpt & $\mathrm{CP}$ diagnosis & $\uparrow$ & [53] \\
\hline \multirow[t]{3}{*}{ Breast cancer } & $\operatorname{IgG~m/z~1591,~} 1794$ & Distinguish stage II patients from NC & $\uparrow$ & [69] \\
\hline & F(6)A4G4 Lac1 & $\begin{array}{l}\text { Poor clinical outcomes in breast cancer, including lymph } \\
\text { node metastasis, recurrent disease, and reduced survival }\end{array}$ & $\uparrow$ & [70] \\
\hline & FUT8 & $\begin{array}{l}\text { Associated with poor prognosis, disease-free survival, } \\
\text { tumour stage and molecular classification }\end{array}$ & $\uparrow$ & {$[21,68]$} \\
\hline \multirow[t]{3}{*}{ Lung cancer } & FUT8 and FUT8 mRNA & $\begin{array}{l}\text { Related to the poor survival rate, tumour stage (N0), male } \\
\text { sex, and disease-free survival of NSCLC patients } \\
\text { Potential plasma biomarker for lung cancer patients }\end{array}$ & $\uparrow$ & $\begin{array}{l}{[21} \\
40-42]\end{array}$ \\
\hline & Core-fucosylated E-cadherin & Prognostic indicator for lung cancer patients & $\uparrow$ & {$[44]$} \\
\hline & $\begin{array}{l}\text { Core fucosylated N-glycans with } \\
\text { additional fucose (Fuc) residue/s }\end{array}$ & Quickly distinguish between cancer and healthy lung tissue & $\uparrow$ & [43] \\
\hline \multirow[t]{3}{*}{$\begin{array}{l}\text { Colorectal cancer } \\
(\mathrm{CRC})\end{array}$} & FUT8 & $\begin{array}{l}\text { As a prognostic marker for stage II and stage III CRC } \\
\text { patients } \\
\text { Related to disease-free survival and microsatellite instability }\end{array}$ & $\uparrow$ & $\begin{array}{l}{[21,46,} \\
47]\end{array}$ \\
\hline & $\begin{array}{l}\text { Core fucosylated bi-antennary glycans } \\
\mathrm{F}(6) \mathrm{A} 2 \mathrm{G} 2 \text { and } \mathrm{F}(6) \mathrm{A} 2 \mathrm{G} 2 \mathrm{~S}(6) 1\end{array}$ & The risk of CRC & $\uparrow$ & [48] \\
\hline & $\begin{array}{l}\text { IgG Core-fucosylation and } \\
\text { sialo Core-fucosylation }\end{array}$ & Possibly related to the development of CRC & $\uparrow$ & [49] \\
\hline $\begin{array}{l}\text { Oral squamous cell } \\
\text { carcinoma (OSCC) }\end{array}$ & Core fucosylated CD147 & Potential marker for the diagnosis of OSCC & $\uparrow$ & {$[60]$} \\
\hline $\begin{array}{l}\text { Epithelial ovarian } \\
\text { cancer (EOC) }\end{array}$ & Core fucosylated CTR1 & $\begin{array}{l}\text { Guiding significance for the identification of patients with } \\
\text { cDDP resistance }\end{array}$ & $\uparrow$ & [71] \\
\hline Gastric cancer (GC) & FUT8 & $\begin{array}{l}\text { Associated with overall survival rate of GC patients in } \\
\text { intestinal-type Lauren classification, male sex and TNM } \\
\text { (stage I-III) }\end{array}$ & $\downarrow$ & $\begin{array}{l}{[21,65,} \\
66]\end{array}$ \\
\hline Melanoma & FUT8 & Diagnose melanoma metastasis & $\uparrow$ & [72] \\
\hline $\begin{array}{l}\text { Oesophageal } \\
\text { squamous cell } \\
\text { carcinoma (ESCC) }\end{array}$ & $\begin{array}{l}\text { FUT8 } \\
\text { Core fucosylated CD147 }\end{array}$ & $\begin{array}{l}\text { A marker to predict the radiotherapy response of ESCC } \\
\text { patients }\end{array}$ & $\uparrow$ & {$[61,62]$} \\
\hline \multirow[t]{2}{*}{ Prostate cancer } & $\begin{array}{l}\text { Core fucosylated prostate-specific antigen } \\
\text { (PSA) }\end{array}$ & $\begin{array}{l}\text { Diagnostic biomarker for differentiating prostate cancer } \\
\text { from other prostate diseases }\end{array}$ & $\uparrow$ & [56] \\
\hline & FUT8 & Prostate cancer aggressiveness & $\uparrow$ & [57] \\
\hline $\begin{array}{l}\text { Papillary thyroid } \\
\text { carcinoma }\end{array}$ & FUT8 & $\begin{array}{l}\text { Differential diagnosis from follicular carcinoma } \\
\text { Related to tumour size and lymph node metastasis }\end{array}$ & $\uparrow$ & {$[63,64]$} \\
\hline $\begin{array}{l}\text { Glioblastoma } \\
\text { multiforme }\end{array}$ & FUT8-AS1 & Associated with poor survival outcomes & $\uparrow$ & {$[21,71]$} \\
\hline Ependymomas & FUT8 & $\begin{array}{l}\text { Related to ependymoma patient age ( } \leq 10 \text { years) and tumour } \\
\text { recurrence status }\end{array}$ & $\uparrow$ & [21] \\
\hline Medulloblastoma & FUT8 & $\begin{array}{l}\text { Associated with tumour stage, metastasis status, molecular } \\
\text { classification and male sex }\end{array}$ & $\uparrow$ & [21] \\
\hline $\begin{array}{l}\text { Diffuse large B-cell } \\
\text { lymphoma }\end{array}$ & FUT8 & Associated with overall survival & $\uparrow$ & [21] \\
\hline
\end{tabular}

Note: $\uparrow$, Increased compared to normal levels; $\downarrow$, decreased compared to normal levels. 
The expression of ceruloplasmin in HCC is higher than that in liver cirrhosis, which is relevant to the diagnostic value of early detection of HCC, and can be used for prognostic evaluation of the survival rate [37]. There are four core fucosylation sites (sites N138, 358, 397, and 762) in ceruloplasmin, and the core fucosylation rate of three sites in alcohol-related HCC was significantly higher than that in alcohol-related cirrhosis [38]. In addition, the core fucosylation of a-1-antitrypsin (A1AT) is an HCC-specific glycoprotein that can predict HCC metastasis [39].

\subsection{FUT8 and core fucosylated E-cadherin as biomarkers for lung cancer}

FUT8 mRNA may serve as a potential plasma biomarker for lung cancer patients [40]. The expression of FUT8 is elevated in non-small-cell lung cancer (NSCLC), and the expression of FUT8 is related to the tumour metastasis, tumour stage (N0), male sex, disease recurrence and a poor survival rate of NSCLC patients [21, 41-42]. Mass spectrometry (MS) analysis of N-glycans compared cancer tissues with adjacent healthy tissues and found that advanced disease stages were mostly observed to have core fucosylated N-glycans with additional fucose (Fuc) residues [43]. In addition, the core fucosylated E-cadherin can be used as a prognostic indicator for lung cancer patients [44], and an AlphaLISA analysis method for quantifying serum core-fucosylated E-cadherin as a biomarker of metastatic lung adenocarcinoma has been developed [45].

\subsection{Diagnostic value of FUT8 and glycoprotein core fucosylation in colorectal cancer}

The expression of E-cadherin and FUT8 are elevated in primary colorectal cancer (CRC) samples [46]. In addition, the increased expression of FUT8 protein can be used as a prognostic marker for stage II and stage III CRC patients and is significantly associated with better disease-free survival (DFS) in p53-negative (but not positive) CRC tumours [47]. And FUT8 expression was also related to microsatellite instability (MSI) [21]. A total of 633 CRC patients and 478 age- and sex- matched controls were analyzed. In all stages of CRC (especially in stage 1), there are statistically significant differences in the plasma N-glycosylation, including the core fucosylated biantennary glycans F(6)A2G2 and $\mathrm{F}(6) A 2 \mathrm{G} 2 \mathrm{~S}(6) 1$, which are significantly reduced [48]. Changes in glycosylation significantly affect the structure and function of immunoglobulin G (IgG), and increased IgG N-glycan core-fucosylation, sialylation and sialo core-fucosylation were are possibly related to the development of CRC [49].

\subsection{FUT8 and glycoprotein core fucosylation for the diagnosis of pancreatic cancer}

Pancreatic ductal adenocarcinoma (PDAC) is the most common type of pancreatic cancer (PC). The expression of FUT8 in PDAC is significantly higher than that in normal pancreatic duct tissue, and is significantly related to lymph node metastasis and recurrence-free survival. In addition, FUT8 gene knockout can significantly reduce the invasion of PDAC cell lines and peritoneal metastasis [50]. Human pancreatic ribonuclease 1 (RNase 1) is a glycoprotein mainly expressed in the pancreas. Although RNase 1 contains similar glycan structures in normal and tumour serum, there was a $40 \%$ increase in core fucosylation in the main sialylated biantennary glycans in the tumor serum RNase 1 [51]. Abnormal mucin overexpression is one of the characteristics of PC. A study found that FUT8 can stabilize the expression of MUC4 and MUC1 at the protein level through core fucosylation [52]. This result suggests that core fucosylated MUC1/4 may also serve as a marker for the occurrence of PC. Chronic pancreatitis (CP) is a common risk factor for PDAC, and total serum core-Fuc-Hpt was shown to be an independent determinant of a subclinical CP diagnosis [53], which is meaningful to distinguish $\mathrm{PDAC}$ and $\mathrm{CP}$ patients.

\subsection{FUT8 and core fucosylated PSA for the diagnosis of prostate cancer}

Distinguishing between prostate cancer and benign prostatic hyperplasia (BPH) is a clinical problem, and finding and applying biomarkers is one of the solutions. Compared with $\mathrm{BPH}$ patients, the expression of core-fucosylated biantennary glycans was significantly increased in prostate cancer patients [54]. Core fucosylated prostate-specific antigen (PSA) may be a diagnostic biomarker for differentiating prostate cancer from other prostate diseases, such as $\mathrm{BPH}$ [55], and both serum and urine tests for core fucosylated PSA are feasible options [56]. In addition, the expression of FUT8 in aggressive tumours (Gleason 8 and above) is higher than that in non-invasive tumours (Gleason 6 and below) [57].

\subsection{FUT8 and core fucosylated CD147 for the diagnosis of oral and oesophageal squamous cell carcinoma}

The survival rates of oral squamous cell carcinoma (OSCC) and oesophageal squamous cell carcinoma (ESCC) are lower than $60 \%$ and $40 \%$, respectively [58]. A late-stage diagnosis and a lack of 
effective treatment strategies make OSCC and ESCC a serious health burden. Compared with normal oral epithelial tissue, the mRNA levels of FUT8 and core glycoprotein in OSCC patients are increased [59]. The application of the glycoproteomics method discovered that core fucosylated CD147 is also considered a potential new tumour marker for the diagnosis of oral cancer [60]. FUT8 expression in ESCC is higher than that in normal oesophageal tissue [61]. FUT8 plays an anti-radiation-driven role in ESCC by core fucosylation of CD147, and it can be used as a marker to predict the radiotherapy response of ESCC patients [62].

\subsection{FUT8 for the diagnosis of papillary thyroid carcinoma}

In papillary thyroid carcinoma, high expression of FUT8 is related to an increased tumour volume and lymph node metastasis [63]. The expression of FUT8 in normal follicles is very low, but FUT8 is highly expressed in $33.3 \%$ of papillary carcinomas, which is directly related to tumour size and lymph node metastasis. In contrast, this phenomenon is relatively rare in follicular carcinoma and anaplastic (undifferentiated) carcinoma, suggesting that the expression of FUT8 may be a key factor in the progression of papillary thyroid carcinoma (rather than follicular carcinoma), and a decrease in FUT8 expression may be related to anaplastic transformation [64].

\subsection{FUT8 for the diagnosis of gastric cancer}

FUT8 is considered to be related to gastric cancer (GC) [65]. Interestingly, unlike other tumours, the expression of FUT8 is decreased in gastric cancer, and the upregulation of FUT8 expression can inhibit the proliferation of human gastric cancer cells [66]. Researchers have shown that the level of core fucosylation residues of at least $9 \mathrm{~N}$-glycan structures in GC is decreased significantly [67]. More importantly, the high expression of FUT8 is related to a better overall survival rate of GC patients along with intestinal-type Lauren classification, male sex and TNM (stage I-III) [21].

\subsection{FUT8 and glycoprotein core fucosylation for the diagnosis of breast cancer}

FUT8 expression was found to be associated with a poor prognosis, advanced tumour stage and type of molecular classification (positive progesterone receptor and oestrogen receptor status), but was less associated with histological grade in breast cancer (BC) [21, 68], and two core fucosylated and agalactosylated glycans (m/z 1591, 1794) in IgG can clearly distinguish stage II patients from NCs [69]. A core-fucosylated tetraantennary $\mathrm{N}$-acetyllactosamine (F(6)A4G4 Lac1) is associated with poor clinical outcomes in BC, including lymph node metastasis, recurrent disease, and reduced survival [70].

\subsection{FUT8 for the diagnosis of glioblastoma multiforme}

Glioblastoma multiforme (GBM) is the most common and deadliest type of primary brain tumours. Although GBM does not generally form distant metastases, the spread of tumour cells to infiltrate the normal cerebral cortex can lead to death. Despite applying a combination of surgical resection, radiotherapy and chemotherapy, the median survival time is only 14 months [71]. FUT8-AS1 is overexpressed in a subgroup of patients with glioblastoma multiforme (GBM) and is associated with even worse poor survival outcomes, information obtained by bioinformatics analysis of TCGA data [72]. A meta-analysis study found that FUT8 expression was less related to pathological typing, but was associated with GBM patient age ( $\leq 40$ years) and a shortened overall survival time of female patients [21].

\subsection{FUT8 for the diagnosis of ependymoma}

Ependymomas mainly involve the supratentorial brain, posterior fossa and spinal cord of the central nervous system, and can occur in both children and adults. At present, the treatment for patients with intracranial ependymoma is still surgical resection combined with radiotherapy, and the survival benefits of chemotherapy for ependymoma are still controversial. At present, there are no conventional molecular or tumour-specific immunohistochemical markers for ependymoma in clinical practice [73]. The discovery of ependymoma diagnostic markers is of great significance for its clinical treatment. The current study found that high expression of FUT8 was related to ependymoma patient age ( $\leq 10$ years) and tumour recurrence status [21]. However, the diagnostic value of FUT8 in ependymomas remains to be discovered.

\subsection{FUT8 for the diagnosis of medulloblastoma}

Medulloblastoma (MB) originates in the brainstem or the part of the cerebellum located in the posterior fossa and is one of the most common malignant brain tumours in childhood [74]. In MB, FUT8 expression levels were found to be associated with tumour stage, metastasis status, molecular classification (WNT and SHH molecular subgroups) and male sex [21]. 


\subsection{FUT8 and glycoprotein core fucosylation for the diagnosis of the other cancers}

The core fucosylation of copper transporter 1 (CTR1) plays an important role in the uptake of cisplatin (cDDP). The level of CTR1 core fucosylation in the serum of cDDP-resistant epithelial ovarian cancer (EOC) patients is significantly increased [75], which has guiding significance for the identification of patients with cDDP resistance. The upregulation of FUT8 and the downregulation of FUT1 and FUT2 were identified as the characteristics of metastatic melanoma [76]. Glycan analysis of total serum reveals increased levels of core fucosylation in cholangiocarcinoma (CCA) [77].

\section{FUT8-mediated EGFR core fucosylation for treatment}

Epidermal growth factor receptor (EGFR) is also known as HER1 or ErbB1 and belongs to the ErbB family of tyrosine kinase receptors along with HER2/neu (ErbB2), HER3 (ErbB3) and HER4 (ErbB4) [78]. EGFR can extensively affect the following downstream signalling pathways: RAS/RAF/MEK/ MAPK/ERK, phosphatidylinositol 3-kinase (PI3K) /Akt, protein kinase C (PKC), Src and JAK/STAT pathways [79]. These signalling cascades can affect cell gene expression, proliferation, angiogenesis, apoptosis inhibition, cell movement, metastasis, adhesion and angiogenesis [80]. Dysregulation of EGFR expression and signalling pathways may play a key role in lung cancer, head and neck squamous cell carcinoma, and colon, pancreas, brain and breast cancer [81], and targeting for EGFR would be a great help to the treatment of these tumours. FUT8 gene knockout in mice resulted in early death, severe growth retardation, and emphysema-like changes in the lung, revealing that FUT8 is essential for the activation of EGFR [82-83].

The EGFR contains $20 \%$ carbohydrates calculated by molecular weight, N-glycosylation affects the structure and stability of this receptor, and the stability and balance of EGFR glycosylation is conducive to cross-cell signal transduction [84-85]. In addition, the $\mathrm{N}$-glycosylation of the extracellular domain of EGFR plays a key role in the binding of growth factors, monoclonal antibodies and EGFR dimers to the extracellular domain of mononuclear EGFR [86]. N-glycosylation is the determinant of EGFR conformation in the cell membrane [87]. These characteristics suggest that targeting EGFR $\mathrm{N}$-glycosylation could be an effective therapy. The expression of FUT8 is related to EGF-mediated activation of JNK or ERK, and the core fucosylation of
$\mathrm{N}$-glycans is necessary for the binding of EGF to the receptor [88]. Modified EGFR core fucosylation increases EGF-mediated cell growth and sensitivity to gefitinib [89]. In contrast to FUT4 and FUT6, FUT8 can promote EGFR dimerization and phosphorylation in lung cancer cells [90]. FUT8 promotes the aggressiveness and malignant TME of non-small cell lung cancer (NSCLC) through EGFR core fucosylation, which accelerates the proliferation of NSCLC cells [91]. Drugs that can reduce EGFR core fucosylation are being used to treat tumours. 2-Fluoro-L-fucose (2FF) treatment reduces the fucosylation level of core membrane glycoproteins, thereby inhibiting downstream signals and inhibiting the progression of liver cancer [92].

In castration-resistant prostate cancer (CRPC), the expression of EGFR and FUT8 has a coordinated regulatory mechanism, and increased expression of FUT8 (because of resistance to androgen deprivation) increases the expression of EGFR; FUT8 plays a key role in converting nuclear receptor signal transduction (androgen receptor) into a cell surface receptor (EGFR) mechanism in prostate cancer cells evading androgen-induced cell death, which leads to an increased survival rate of prostate cancer cells under hormone depletion conditions [93]. FUT8 silencing reduces the growth rate of TGP49 mouse pancreatic acinar cell carcinoma by attenuating EGFR phosphorylation and the EGFR-trypsin-PAR-2 pathway [94]. FUT8 (-/-)) mice were treated with diethylnitrosamine (DEN) and pentobarbital (PB). In FUT8 (+/+) and FUT8 (+/-) mice, after DEN and PB treatment, the expression of FUT8 was increased, which induced multiple large, vascularized nodules. However, the formation of HCC in FUT8 (-/-) mice was almost completely inhibited. The inhibitory effect of FUT8 deficiency on tumorigenesis was also confirmed by the disappearance of tumour formation by human hepatoma cell line cells lacking FUT8 in xenograft tumour models. In addition, the deletion of the FUT8 gene leads to a weakened response of the HepG2 cell line to epidermal growth factor (EGF) and hepatocyte growth factor (HGF), which provides a possible mechanism by which FUT8 participates in liver cancer [95]. In summary, the core glycosylation mediated by FUT8 has a significant impact on EGFR signalling and is a therapeutic target for cancer.

\section{FUT8-mediated TGFBR core fucosylation for treatment}

TGF- $\beta 1,2$ and 3 are receptor ligands with similar biological activity, and are important in regulating proliferation, migration, differentiation, and apoptosis. There are also three TGF- $\beta$ receptors named TGFBR1, 2 , and 3. Canonical TGF- $\beta$ signalling 
occurs when TGF- $\beta 1,2$ or 3 binds to TGFBR2, and then TGFBR1 is recruited and phosphorylated. After that, phosphorylated TGFBR1 increases SMAD2/3 phosphorylation, and then activated SMAD2/3 can form a complex with a common SMAD mediator, i.e., SMAD4, and translocate into the nucleus where the heteromeric complex modulates the transcription of target genes, which then recruit SMAD4 and translocate to the nucleus where it regulates the transcription of TGF- $\beta$ target genes [96-98]. Both receptors are $\mathrm{N}$-glycoproteins with potential $\mathrm{N}$-linked glycosites at Asn-70, 94, and 154 of TGFBR2 and at Asn-45 of TGFBR1, and TGF- $\beta$ sensitivity can be regulated by $\mathrm{N}$-linked glycosylation of TGFBR [99-100].

A recent study showed that two conserved asparagine residues, Asn-70 and 94, are $\mathrm{N}$-glycosylated and are essential for the cell-surface transport of TGFBR2 [99]. First, core fucosylation may cause conformational alterations of TGF- $\beta$ receptors. In addition, TGFBR $\mathrm{N}$-glycosylation is necessary for its transport on the cell surface [101]. In the TGF- $\beta /$ Smad $2 / 3$ signalling pathway, silencing FUT8 did not inhibit the protein expression of the TGF- $\beta$ receptor and Smad $2 / 3$ protein; but it did significantly inhibited the phosphorylation of $\operatorname{Smad} 2 / 3$ and epithelial-mesenchymal transition (EMT). Similarly, in PDGF/ERK signal transduction, silencing FUT8 did not affect the expression of platelet-derived growth factor (PDGF) receptor and ERK protein, but it did significantly inhibit ERK phosphorylation and EMT [102]. More importantly, even if the expression level of TGFBR increases, blocking core fucosylation can also weaken EMT [103]. In addition, FUT8 is highly upregulated during the process of EMT induced by TGF- $\beta$, FUT8 overexpression stimulates EMT, and silencing FUT8 inhibits tumour invasion and migration [104]. The core fucosylation of $\mathrm{N}$-glycan in TGFBR is necessary for receptor function [105]. Preventing FUT8-mediated TGFBR core fucosylation is of great significance for tumour EMT suppression, which is an important target for interference with EMT. Intervening in TGFBR core fucosylation is a feasible solution for inhibiting tumour EMT.

\section{FUT8-mediated E-cadherin core fucosylation for treatment}

E-cadherin belongs to the type-I cadherin family and is generally considered to be the prototype of all cadherins [106]. Decreased expression of E-cadherin is a recurring finding in cancer, leading to tumour cell proliferation, survival, invasion and loss of epithelial cell polarity [107]. The polysaccharide structure of E-cadherin has an important influence on its function.
E-cadherin O-glycosylation in the cytoplasm blocks E-cadherin transport during the process of apoptosis, leading to decreased intercellular adhesion and endoplasmic reticulum stress [108]. The $\mathrm{N}$-glycosylation site of E-cadherin located in extracellular region 4 (EC4) changes the molecular structure of E-cadherin, thereby destabilizing adherens junctions [109]. In addition, there are two $\mathrm{N}$-glycosylation sites on E-cadherin located in EC5 [109]. The N-glycan on Asn 633 is essential for the folding, transport and correct expression of E-cadherin [110]. The degree of E-cadherin N-glycan branching degree is inversely related to the establishment of cell adhesion [111]. In fact, E-cadherin N-glycosylation is more common than the other two known E-cadherin posttranslational modifications [107]. $\mathrm{N}$-acetylglucosamine transferase III (GnT-III), N-acetylglucosamine transferase V (GnT-V) and FUT8 play important roles in N-glycan remodelling of E-cadherin [112]. Therefore, FUT8-mediated E-cadherin core fucosylation may have an important impact on tumor cell the malignant phenotype of tumour cells.

FUT8 plays an important role in the E-cadherin redistribution and downstream signal regulation. Core fucosylation of E-cadherin could significantly hinder the three-dimensional conformation effect of $\mathrm{N}$-glycan on E-cadherin, resulting in conformational asymmetry, thereby inhibiting the function of E-cadherin [43]. In colon cancer WiDr cells transfected with FUT8, FUT8 participates in the emergence of a low-molecular-weight E-cadherin population, regulates the total amount of Ecadherin, regulates the transfer potential and the stability of cell-to-cell contact [46]. The lack of FUT8 inhibits the FAK/integrin pathway by inhibiting E-cadherin core fucosylation, reducing the accumulation of nuclear chain proteins and inhibiting the expression of MMP-2 and MMP-9, which inhibits the adhesion, migration and invasion of breast cancer cells [112]. In addition, activation of the Wnt/ $\beta$-catenin signalling pathway upregulates the expression level of FUT8, thereby inducing an increase in stemness and EMT in breast cancer cells [113]. In human low and highly metastatic giant lung cancer lines (95C, 95D), core-fucosylated E-cadherin regulates nuclear $\beta$-catenin accumulation [114], and Src activation and induces epithelial-mesenchymal transition (EMT)-like processes [115]. Deoxycholic acid impairs glycosylation and core fucosylation processes in oesophageal epithelial cells, reducing the expression of E-cadherin [116]. Therefore, intervention into FUT8-mediated E-cadherin core fucosylation in tumours is of great significance for E-cadherin loss and relocation. 


\section{FUT8-mediated PD-1/PD-L1 core fucosylation for treatment}

Tumour cells can evade T-cell-mediated cytotoxicity by inhibiting programmed cell death protein 1 (PD-1)/programmed cell death ligand 1 (PD-L1) immune checkpoints [117]. Clinical trials have shown that anti-PD-1/PD-L1 monoclonal antibodies are effective in treating melanoma, non-small-cell lung cancer (NSCLC), renal cell carcinoma (RCC), and bladder cancer [118]. In fact, both PD-1 (NM-005018) and PD-L1 (NM-001267706) are glycoproteins with four core-fucosylated N-glycans, namely, N4, 49, 58, 116 and N35, 192, 200, 219, respectively [119-120]. Glycosylation of PD-1, especially at the N58 site, plays a crucial role in mediating its interaction with PD-L1 [121]. PD-L1 N-glycosylation sites at N192, N200 and N219 antagonize GSK3 $\beta$ binding and decrease the phosphorylation-dependent proteasomal degradation of PD-L1 by $\beta-\operatorname{TrCP}$ [122]. Inhibition of the core transferase FUT8 can reduce the expression of PD-1 on the cell surface, enhance the activation of $\mathrm{T}$ cells, and thus more effectively eradicate the tumours [123]. The absence of FUT8 significantly enhanced the ubiquitination of PD-1, leading to the degradation of PD-1 in the proteasome [124]. Therefore, interference into PD-1/PD-L1 core fucosylation mediated by FUT8 is valuable for blocking PD-1/PD-L1-mediated tumour immune escape.

\section{Core fucosylated $\alpha 3 \beta 1$ integrin for treatment}

Integrins belong to the family of cell-adhesion molecules (CAMs), which are include two noncovalently linked heterodimer $\alpha$ and $\beta$ subunits, mediate cell-to-cell and cell-to-extracellular matrix (ECM) adhesion, and provide adhesion and traction during cell movement [125-126]. In recent years, abnormal expression of integrin has been found in many tumours, and it has an impact on the differentiation, migration, proliferation and angiogenesis of tumour cells. In addition, research on integrin-targeted drugs is of great significance for the clinical treatment of tumours [127]. The activity of integrins is strongly influenced by glycosylation events and glycan-mediated interactions [128]. Integrins contain more than 20 potential glycosylation sites [129]. The existence of an N-glycan structure is essential for integrin heterodimerization, conformational stability, expression on the cell membrane, and interaction with ligands [128]. $\mathrm{N}$-glycosylation is dynamic. The remodelling of $\mathrm{N}$-glycosyls due to the action of glycosyltransferase can regulate the binding of integrins to substrates and play a key role in cell adhesion and migration [130]. FUT8 is essential for a $3 \beta 1$ integrin core fucosylation. FUT8-mediated a3 $\beta 1$ integrin core fucosylation can induce laminin 5-induced migration and intracellular signal transduction [131]. N-glycan core fucosylation on the cell surface acceptor can promote the self-assembly of cancer cells mediated by cyclo-RGDfK (TPP) to form three-dimensional multicellular tumour spheroids [132].

\section{Defucosylated IGg1 improves treatment performance}

Monoclonal antibody therapy is increasingly used to treat cancer, and market data predict that the growth rate of monoclonal antibodies will be higher than any other therapeutic biological drug [133]. Most therapeutic antibodies are of the human IgG1 subtype. Complement-dependent cytotoxicity and antibody-dependent cytotoxicity (ADCC) are the main mechanisms by which antibodies activate the immune system [134]. Natural killer cells activate ADCC mainly by binding to the Fc $\gamma$ RIIIa receptor via the FC part of the antibody [135]. The effect of natural killer (NK) cell recruitment and activation is determined by the affinity of IgG to FcyRIIIa, which is related to IGg FC oligosaccharide core fucosylation [136-137]. Core fucosylation at asparagine 297 decreased ADCC activities by weakening its binding affinity to FcyRIIIa [138].

Mogamulizumab (POTELIGEO ${ }^{\circledR}$ ) was the first glycoengineered antibody to reach the market; it is a defucosylated, humanized, anti-chemokine receptor 4 $\mathrm{mAb}$ and is considered a milestone for therapeutic antibodies [139]. Obinutuzumab (GA101 or Gazyva ${ }^{\circledR}$ ) is the first glycoengineered therapeutic anti-CD20 antibody approved by the FDA in 2013 for the combined treatment of chronic lymphocytic leukaemia (CLL) and follicular lymphoma patients [140]. A phase $1 / 2$ GAUGUIN study showed that obinutuzumab monotherapy is effective in CLL and non-Hodgkin lymphoma patients with relapse/refractoriness [141-142]. In phase 3 clinical trials, GA101 combined with chemotherapy can significantly prolonged overall survival and progression-free survival and improved complete remission rates in CLL patients [143].

Chinese hamster ovary $(\mathrm{CHO})$ cells are used to produce $35.5 \%$ of all launched therapeutic antibodies [144], but the IgG1 antibody produced by $\mathrm{CHO}$ is extensively core fucosylated [145]. After disrupting the FUT8 allele in the CHO/DG44 cell line, produced anti-CD20 IgG1 strongly binds to human FcRIIIa and dramatically enhances ADCC to approximately 100-fold higher than that of rituxan [146]. In addition, the growth of FUT8 knockout CHO-S (FUT8 -/-) cells 
is similar to that of wild-type CHO-S cells [147] and antibodies produced by the FUT8 (-/-) CHO cell line without adverse phenotypic effects [145].

\section{Conclusion and prospect}

FUT8 and core fucosylated glycoproteins are frequently upregulated in human tumours and may be used as markers for tumour diagnosis (Table 1). In contrast, the expression of FUT8 is downregulated in gastric cancer, and the upregulation of FUT8 is associated with a better overall survival rate of GC patients [21]. The functional implications of low FUT8 in gastric cancer have not been well studied. The core fucosylation changes of the $\mathrm{N}$-glycan structure of some important receptors in gastric cancer may regulate signal transduction [66]. In recent decades, new technologies such as MALDI mass spectrometry glycan imaging have been used to identify specific glycan changes in cancer tissues [148]. Core fucosylated $\mathrm{N}$-glycan structures have great potential to serve as cancer diagnosis, prognosis, and treatment monitoring biomarkers.

The significance of FUT8 expression changes in human tumours is still not fully elucidated, even though the existing studies have proved that the upregulation of FUT8 is prevalent in most human tumors. New fucosylation inhibitors (SGN-2FF, A2FF1P, B2FF1P, P-D-Rha6F2-1P and P-D-Rha6F3-1P) have been developed [20], but no evidence indicates that they are specific inhibitors that inhibit the synthesis of a-1,6 fucoside bonds. In some tumours, such as HNSCC and lung cancer, inhibiting the synthesis of $a-1,3 / 4$ fucoside bonds may promote tumour progression by improving EGFR activity [149-150]. However, it should be noted that the inhibition of FUT8 may considerably impact normal human cells, thus limiting the application of FUT8 inhibitors in tumour therapy $[149,151]$. Therefore, targeted therapy of key core fucosylated glycoproteins, such as the core fucosylation sites of EGFR, TGFBR, E-cadherin, PD-1/PDL-1 and a3 $\beta 1$ integrin, may be a more feasible approach.

\section{Acknowledgements}

This study was supported by the Outstanding Young Talent Project of Zunyi Medical University (17zy-002) and PhD Research Fund of Zunyi medical university (F-801); Top Science and Technology Talent Project in Higher Education Institution of Guizhou Province (grant No. Qian-Jiao-He KY Zi [2016]080); the sixth talent foundation in Guizhou province $<$ Guizhou province talent foundation --- Research and development of medical biological materials> (rcjd2019-9); Guizhou Provincial Department of Education Youth Science and Technology Talents
Growth Project (QIAN-JIAO-HE KY ZI [2018]236); Guizhou Provincial Graduate Research Fund (QIAN-JIAO-HE YJSCXJH [2019]087).

\section{Author Contributions}

$\mathrm{C}, \mathrm{C}, \mathrm{L}$ wrote the manuscript. $\mathrm{Q}, \mathrm{W}$ primarily revised and finalized manuscript. J,X,A design and produce table and figure. $\mathrm{S}, \mathrm{Q}, \mathrm{Y} \mathrm{Z}, \mathrm{X}, \mathrm{T} \mathrm{H}, \mathrm{W}$ and $\mathrm{H}, \mathrm{L}$ collect the literature. $X, Y, G$ and J,G,L fix language and syntax errors.

\section{Competing Interests}

The authors have declared that no competing interest exists.

\section{References}

1. Pinho SS, Reis CA. Glycosylation in cancer: mechanisms and clinical implications. Nat Rev Cancer. 2015; 15(9): 540-55.

2. Taniguchi N, Honke K, Fukuda M, et al. Handbook of Glycosyltransferases and Related Genes. Springer Japan. 2014.

3. Narimatsu H. Human glycogene cloning: focus on beta 3-glycosyltransferase and beta 4-glycosyltransferase families. Curr Opin Struct Biol. 2006; 16(5): 567-75.

4. Lowe JB, Marth JD. A genetic approach to Mammalian glycan function. Annu Rev Biochem. 2003; 72: 643-91.

5. Taniguchi N, Miyoshi E, Gu J, et al. Decoding sugar functions by identifying target glycoproteins. Curr Opin Struct Biol. 2006; 16(5): 561-6.

6. Tu Z, Lin $\mathrm{YN}$, Lin $\mathrm{CH}$. Development of fucosyltransferase and fucosidase inhibitors. Chem Soc Rev. 2013; 42(10): 4459-75.

7. Mollicone R, Moore SE, Bovin N, et al. Activity, splice variants, conserved peptide motifs, and phylogeny of two new alpha1,3-fucosyltransferase families (FUT10 and FUT11). J Biol Chem. 2009; 284(7): 4723-38.

8. Luo Y, Nita-Lazar A, Haltiwanger RS. Two distinct pathways for O-fucosylation of epidermal growth factor-like or thrombospondin type 1 repeats. J Biol Chem. 2006; 281(14): 9385-92.

9. Schneider M, Al-Shareffi E, Haltiwanger RS. Biological functions of fucose in mammals. Glycobiology. 2017; 27(7): 601-18.

10. Taniguchi N, Korekane H. Branched N-glycans and their implications for cell adhesion, signaling and clinical applications for cancer biomarkers and in therapeutics. BMB Rep. 2011; 44(12): 772-81.

11. Yamaguchi $\mathrm{Y}$, Fujii $\mathrm{J}$, Inoue $\mathrm{S}$, et al. Mapping of the alpha-1,6-fucosyltransferase gene, FUT8, to human chromosome 14q24.3. Cytogenet Cell Genet. 1999; 84(1-2): 58-60.

12. Miyoshi E, Moriwaki K, Nakagawa T. Biological function of fucosylation in cancer biology. J Biochem. 2008; 143(6): 725-29.

13. Ihara H, Ikeda $Y$, Taniguchi N. Reaction mechanism and substrate specificity for nucleotide sugar of mammalian alpha1,6-fucosyltransferase--a large-scale preparation and characterization of recombinant human FUT8. Glycobiology. $2006 ; 16(4): 333-42$

14. Uozumi N, Yanagidani S, Miyoshi E, et al. Purification and cDNA cloning of porcine brain GDP-L-Fuc:N-acetyl-beta-D-glucosaminide alpha1--> 6fucosyltransferase. J Biol Chem. 1996; 271(44): 27810-7.

15. Zafra-Ruano A, Luque I. Interfacial water molecules in $\mathrm{SH} 3$ interactions: Getting the full picture on polyproline recognition by protein-protein interaction domains. FEBS Lett. 2012; 586(17): 2619-30.

16. Tomida S, Takata M, Hirata T, et al. The SH3 domain in the fucosyltransferase FUT8 controls FUT8 activity and localization and is essential for core fucosylation. J Biol Chem. 2020; 295(23): 7992-8004.

17. García-García A, Ceballos-Laita L, Serna S, et al. Structural basis for substrate specificity and catalysis of a1,6-fucosyltransferase. Nat Commun. 2020; 11(1): 973.

18. Calderon AD, Liu Y, Li X, et al. Substrate specificity of FUT8 and chemoenzymatic synthesis of core-fucosylated asymmetric N-glycans. Org Biomol Chem. 2016; 14(17): 4027-31.

19. Järvå MA, Dramicanin M, Lingford JP, et al. Structural basis of substrate recognition and catalysis by fucosyltransferase 8. J Biol Chem. 2020; 295(19): 6677-88.

20. Bastian K, Scott E, Elliott DJ, et al. FUT8 Alpha-(1,6)-Fucosyltransferase in Cancer. Int J Mol Sci. 2021; 22(1): 455.

21. Taniguchi N, Kizuka Y. Glycans and cancer: role of N-glycans in cancer biomarker, progression and metastasis, and therapeutics. Adv Cancer Res. 2015; 126: 11-51.

22. Ma M, Han G, Wang Y, et al. Role of FUT8 expression in clinicopathology and patient survival for various malignant tumor types: a systematic review and meta-analysis. Aging (Albany NY). 2020; 13(2): 2212-30. 
23. Luo P, Yin P, Hua R, et al. A Large-scale, multicenter serum metabolite biomarker identification study for the early detection of hepatocellular carcinoma. Hepatology. 2018; 67(2): 662-75.

24. Chalasani NP, Ramasubramanian TS, Bhattacharya A, et al. A Novel Blood-based Panel of Methylated DNA and Protein Markers for Detection of Early-Stage Hepatocellular Carcinoma. Clin Gastroenterol Hepatol. 2020; Sep 1: S1542-3565(20)31224-6.

25. Wongjarupong N, Negron-Ocasio GM, Mara KC, et al. BALAD and BALAD-2 predict survival of hepatocellular carcinoma patients: a North American cohort study. HPB (Oxford). 2020: S1365-182X(20)31159-X.

26. Sumida Y, Yoneda M, Seko Y, et al. Surveillance of Hepatocellular Carcinoma in Nonalcoholic Fatty Liver Disease. Diagnostics (Basel). 2020; 10(8): 579.

27. Hameed B, Mehta N, Sapisochin G, et al. Alpha-fetoprotein level > 1000 $\mathrm{ng} / \mathrm{mL}$ as an exclusion criterion for liver transplantation in patients with hepatocellular carcinoma meeting the Milan criteria. Liver Transpl. 2014; 20(8): 945-51.

28. Chen S, Li J, Tan X, et al. Clinical role of combining alpha-fetoprotein and lens culinaris agglutinin-reactive fraction of alpha-fetoprotein for hepatocellular carcinoma: Evidence from literature and an original study. J Clin Lab Anal. 2020; 34(7): e23262.

29. Uozumi N, Yanagidani S, Miyoshi E, et al. Purification and cDNA cloning of porcine brain GDP-L-Fuc:N-acetyl-beta-D-glucosaminide alpha1--> 6fucosyltransferase. J Biol Chem. 1996; 271(44): 27810-7.

30. Cheng L, Gao S, Song X, et al. Comprehensive N-glycan profiles of hepatocellular carcinoma reveal association of fucosylation with tumor progression and regulation of FUT8 by microRNAs. Oncotarget. 2016; 7(38): 61199-214.

31. Zhang $\mathrm{C}, \mathrm{Wu} \mathrm{Q}$, Huang $\mathrm{H}$, et al. Caveolin-1 upregulates FUT8 expression by activating the Wnt/ $\beta$-catenin pathway to enhance HCC cell proliferative and invasive ability. Cell Biol Int. 2020; 44(11): 2202-12.

32. Cheng L, Luo S, Jin C, et al. FUT family mediates the multidrug resistance of human hepatocellular carcinoma via the PI3K/Akt signaling pathway. Cell Death Dis. 2013; 4(11): e923.

33. Zhao T, Jia L, Li J, et al. Heterogeneities of Site-Specific N-Glycosylation in HCC Tumors With Low and High AFP Concentrations. Front Oncol. 2020; 10: 496.

34. Di Masi A, De Simone G, Ciaccio C, et al. Haptoglobin: From hemoglobin scavenging to human health. Mol Aspects Med. 2020; 73: 100851

35. Zhu J, Lin Z, Wu J, et al. Analysis of serum haptoglobin fucosylation in hepatocellular carcinoma and liver cirrhosis of different etiologies. J Proteome Res. 2014; 13(6): 2986-97.

36. Pompach P, Ashline DJ, Brnakova Z, et al. Protein and site specificity of fucosylation in liver-secreted glycoproteins. J Proteome Res. 2014; 13(12): 5561-9.

37. Casaril M, Capra F, Marchiori L, et al. Serum copper and ceruloplasmin in early and in advanced hepatocellular carcinoma: diagnostic and prognostic relevance. Tumori. 1989; 75(5): 498-502.

38. Yin $\mathrm{H}$, Lin Z, Nie S, et al. Mass-selected site-specific core-fucosylation of ceruloplasmin in alcohol-related hepatocellular carcinoma. J Proteome Res. 2014; 13(6): 2887-96.

39. Comunale MA, Rodemich-Betesh L, Hafner J, et al. Linkage specific fucosylation of alpha-1-antitrypsin in liver cirrhosis and cancer patients: implications for a biomarker of hepatocellular carcinoma. PLoS One. 2010; 5(8): e12419.

40. Leng $\mathrm{Q}$, Lin $\mathrm{Y}, \mathrm{Zhan} \mathrm{M}$, et al. An integromic signature for lung cancer early detection. Oncotarget. 2018; 9(37): 24684-92.

41. Park S, Lim JM, Chun JN, et al. Altered expression of fucosylation pathway genes is associated with poor prognosis and tumor metastasis in non-small cell lung cancer. Int J Oncol. 2020; 56(2): 559-67.

42. Chen $\mathrm{CY}$, Jan $\mathrm{YH}$, Juan $\mathrm{YH}$, et al. Fucosyltransferase 8 as a functional regulator of nonsmall cell lung cancer. Proc Natl Acad Sci U S A. 2013; 110(2): 630-5.

43. Lattová E, Skřičková J, Hausnerová J, et al. N-Glycan profiling of lung adenocarcinoma in patients at different stages of disease. Mod Pathol. 2020; 33(6): 1146-56

44. Geng F, Shi BZ, Yuan YF, et al. The expression of core fucosylated E-cadherin in cancer cells and lung cancer patients: prognostic implications. Cell Res. 2004; 14(5): 423-33.

45. Wen CL, Chen KY, Chen CT, et al. Development of an AlphaLISA assay to quantify serum core-fucosylated E-cadherin as a metastatic lung adenocarcinoma biomarker. J Proteomics. 2012;75(13): 3963-76.

46. Osumi D, Takahashi M, Miyoshi E, et al. Core fucosylation of E-cadherin enhances cell-cell adhesion in human colon carcinoma WiDr cells. Cancer Sci. 2009; 100(5): 888-95.

47. Noda M, Okayama H, Kofunato Y, et al. Prognostic role of FUT8 expression in relation to p53 status in stage II and III colorectal cancer. PLoS One. 2018; 13(7): e0200315.

48. Doherty M, Theodoratou E, Walsh I, et al. Plasma N-glycans in colorectal cancer risk. Sci Rep. 2018; 8(1): 8655.

49. Liu S, Cheng L, Fu Y, et al. Characterization of IgG N-glycome profile in colorectal cancer progression by MALDI-TOF-MS. J Proteomics. 2018; 181: 225-37.

50. Tada K, Ohta M, Hidano S, et al. Fucosyltransferase 8 plays a crucial role in the invasion and metastasis of pancreatic ductal adenocarcinoma. Surg Today. 2020; 50(7): 767-77
51. Barrabés S, Pagès-Pons L, Radcliffe CM, et al. Glycosylation of serum ribonuclease 1 indicates a major endothelial origin and reveals an increase in core fucosylation in pancreatic cancer. Glycobiology. 2007; 17(4): 388-400.

52. Kumar S, Das S, Rachagani S, et al. NCOA3-mediated upregulation of mucin expression via transcriptional and post-translational changes during the development of pancreatic cancer. Oncogene. 2015; 34(37): 4879-89.

53. Ueda M, Kamada Y, Takamatsu S, et al. Specific increase in serum core-fucosylated haptoglobin in patients with chronic pancreatitis. Pancreatology. 2016; 16(2): 238-43.

54. Saldova R, Fan Y, Fitzpatrick JM, et al. Core fucosylation and alpha2-3 sialylation in serum $\mathrm{N}$-glycome is significantly increased in prostate cancer comparing to benign prostate hyperplasia. Glycobiology. 2011; 21(2): 195-205.

55. Lang R, Leinenbach A, Karl J, et al. An endoglycosidase-assisted LC-MS/MS-based strategy for the analysis of site-specific core-fucosylation of low-concentrated glycoproteins in human serum using prostate-specific antigen (PSA) as example. Clin Chim Acta. 2018; 480: 1-8.

56. Kammeijer GSM, Nouta J, de la Rosette JJMCH, et al. An In-Depth Glycosylation Assay for Urinary Prostate-Specific Antigen. Anal Chem. 2018; 90(7): 4414-21.

57. Wang X, Chen J, Li QK, et al. Overexpression of a $(1,6)$ fucosyltransferase associated with aggressive prostate cancer. Glycobiology. 2014; 24(10): 935-44.

58. Khan T, Relitti N, Brindisi M, et al. Autophagy modulators for the treatment of oral and esophageal squamous cell carcinomas. Med Res Rev. 2020 May; 40(3):1002-60.

59. Chang SC, Lin WL, Chang YF, et al. Glycoproteomic identification of novel plasma biomarkers for oral cancer. J Food Drug Anal. 2019; 27(2): 483-93.

60. Chen YT, Chong YM, Cheng CW, et al. Identification of novel tumor markers for oral squamous cell carcinoma using glycoproteomic analysis. Clin Chim Acta. 2013; 420: 45-53.

61. Sadeghzadeh Z, Khosravi A, Jazi MS, et al. Upregulation of Fucosyltransferase 3,8 and protein O-Fucosyltransferase 1,2 genes in esophageal cancer stem-like cells (CSLCs). Glycoconj J. 2020; 37(3): 319-27.

62. Shen L, Xia M, Deng X, et al. A lectin-based glycomic approach identifies FUT8 as a driver of radioresistance in oesophageal squamous cell carcinoma. Cell Oncol (Dordr). 2020; 43(4): 695-707.

63. Miyoshi E, Ito Y, Miyoshi Y. Involvement of aberrant glycosylation in thyroid cancer. J Oncol. 2010; 2010: 816595.

64. Ito $\mathrm{Y}$, Miyauchi A, Yoshida H, et al. Expression of alpha1,6-fucosyltransferase (FUT8) in papillary carcinoma of the thyroid: its linkage to biological aggressiveness and anaplastic transformation. Cancer Lett. 2003; 200(2): $167-72$.

65. Wu J, Xiao Y, Xia C, et al. Identification of Biomarkers for Predicting Lymph Node Metastasis of Stomach Cancer Using Clinical DNA Methylation Data. Dis Markers. 2017; 2017: 5745724

66. Zhao YP, Xu XY, Fang M, et al. Decreased core-fucosylation contributes to malignancy in gastric cancer. PLoS One. 2014; 9(4): e94536.

67. Liu L, Yan B, Huang J, et al. The identification and characterization of novel N-glycan-based biomarkers in gastric cancer. PLoS One. 2013; 8(10): e77821.

68. Yue L, Han C, Li Z, et al. Fucosyltransferase 8 expression in breast cancer patients: A high throughput tissue microarray analysis. Histol Histopathol. 2016; 31(5): 547-55

69. Gemechu T, Lako W, Hinou $\mathrm{H}$, et al. Exploring serum and immunoglobulin $\mathrm{G}$ $\mathrm{N}-$ glycome as diagnostic biomarkers for early detection of breast cancer in Ethiopian women. BMC Cancer. 2019; 19(1): 588.

70. Herrera $\mathrm{H}$, Dilday $\mathrm{T}$, Uber $\mathrm{A}$, et al. Core-Fucosylated Tetra-Antennary N-Glycan Containing A Single N-Acetyllactosamine Branch Is Associated with Poor Survival Outcome in Breast Cancer. Int J Mol Sci. 2019; 20(10): 2528.

71. Kotliarova S, Fine HA. SnapShot: glioblastoma multiforme. Cancer Cell. 2012; 21(5): 710-710.e1.

72. Shergalis A, Bankhead A 3rd, Luesakul U, et al. Current Challenges and Opportunities in Treating Glioblastoma. Pharmacol Rev. 2018; 70(3): 412-45.

73. Neumann JE, Spohn M, Obrecht D, et al. Molecular characterization of histopathological ependymoma variants. Acta Neuropathol. 2020; 139(2): 305-18.

74. Gajjar AJ, Robinson GW. Medulloblastoma-translating discoveries from the bench to the bedside. Nat Rev Clin Oncol. 2014; 11(12): 714-22.

75. Lv X, Song J, Xue K, et al. Core fucosylation of copper transporter 1 plays a crucial role in cisplatin-resistance of epithelial ovarian cancer by regulating drug uptake. Mol Carcinog. 2019; 58(5): 794-807.

76. Agrawal P, Fontanals-Cirera B, Sokolova E, et al. A Systems Biology Approach Identifies FUT8 as a Driver of Melanoma Metastasis. Cancer Cell. 2017; 31(6): 804-19.

77. Betesh L, Comunale MA, Wang M, et al. Identification of fucosylated Fetuin-A as a potential biomarker for cholangiocarcinoma. Proteomics Clin Appl. 2017; 11(9-10): 10.1002/prca.201600141.

78. COHEN S. Isolation of a mouse submaxillary gland protein accelerating incisor eruption and eyelid opening in the new-born animal. J Biol Chem. 1962; 237: 1555-62.

79. Chong CR, Jänne PA. The quest to overcome resistance to EGFR-targeted therapies in cancer. Nat Med. 2013; 19(11): 1389-400.

80. Goldman CK, Kim J, Wong WL, et al. Epidermal growth factor stimulates vascular endothelial growth factor production by human malignant glioma cells: a model of glioblastoma multiforme pathophysiology. Mol Biol Cell. 1993; 4(1): 121-33. 
81. Brand TM, Iida M, Luthar $\mathrm{N}$, et al. Nuclear EGFR as a molecular target in cancer. Radiother Oncol. 2013; 108(3): 370-7.

82. Wang $\mathrm{X}, \mathrm{Gu} \mathrm{J}$, Ihara $\mathrm{H}$, et al. Core fucosylation regulates epidermal growth factor receptor-mediated intracellular signaling. J Biol Chem. 2006; 281(5): $2572-7$

83. Wang $\mathrm{X}$, Inoue $\mathrm{S}, \mathrm{Gu} \mathrm{J}$, et al. Dysregulation of TGF-beta1 receptor activation leads to abnormal lung development and emphysema-like phenotype in core fucose-deficient mice. Proc Natl Acad Sci U S A. 2005; 102(44): 15791-6.

84. Cummings RD, Soderquist AM, Carpenter G. The oligosaccharide moieties of the epidermal growth factor receptor in A-431 cells. Presence of complex-type $\mathrm{N}$-linked chains that contain terminal $\mathrm{N}$-acetylgalactosamine residues. J Biol Chem. 1985; 260(22): 11944-52.

85. Whitson KB, Whitson SR, Red-Brewer ML, et al. Functional effects of glycosylation at Asn-579 of the epidermal growth factor receptor. Biochemistry. 2005; 44(45): 14920-31.

86. Azimzadeh Irani M, Kannan S, Verma C. Role of N-glycosylation in EGFR ectodomain ligand binding. Proteins. 2017; 85(8): 1529-49.

87. Kaszuba K, Grzybek M, Orłowski A, et al. N-Glycosylation as determinant of epidermal growth factor receptor conformation in membranes. Proc Natl Acad Sci U S A. 2015; 112(14): 4334-9.

88. Wang $\mathrm{X}, \mathrm{Gu}$ J, Ihara $\mathrm{H}$, et al. Core fucosylation regulates epidermal growth factor receptor-mediated intracellular signaling. J Biol Chem. 2006; 281(5): 2572-7.

89. Matsumoto K, Yokote H, Arao T, et al. N-Glycan fucosylation of epidermal growth factor receptor modulates receptor activity and sensitivity to epidermal growth factor receptor tyrosine kinase inhibitor. Cancer Sci. 2008; 99(8): 1611-7.

90. Hsu TL, Wong CH. Sialylation and fucosylation of epidermal growth factor receptor suppress its dimerization and activation in lung cancer cells. Proc Natl Acad Sci U S A. 2011; 108(28): 11332-7.

91. Li $\mathrm{F}$, Zhao $\mathrm{S}$, Cui $\mathrm{Y}$, et al. a1,6-Fucosyltransferase (FUT8) regulates the cancer-promoting capacity of cancer-associated fibroblasts (CAFs) by modifying EGFR core fucosylation (CF) in non-small cell lung cancer (NSCLC). Am J Cancer Res. 2020; 10(3): 816-37.

92. Zhou Y, Fukuda T, Hang Q, et al. Inhibition of fucosylation by 2-fluorofucose suppresses human liver cancer HepG2 cell proliferation and migration as well as tumor formation. Sci Rep. 2017; 7(1): 11563.

93. Höti N, Lih TS, Pan J, et al, A Comprehensive Analysis of FUT8 Overexpressing Prostate Cancer Cells Reveals the Role of EGFR in Castration Resistance. Cancers (Basel). 2020; 12(2): 468.

94. Li W, Nakagawa T, Koyama N, et al. Down-regulation of trypsinogen expression is associated with growth retardation in alpha1,6-fucosyltransferase-deficient mice: attenuation of proteinase-activated receptor 2 activity. Glycobiology. 2006; 16(10): 1007-19.

95. Wang $Y$, Fukuda $T$, Isaji $T$, et al. Loss of a1,6-fucosyltransferase inhibits chemical-induced hepatocellular carcinoma and tumorigenesis by down-regulating several cell signaling pathways. FASEB J. 2015; 29(8): 3217-27.

96. Hata A, Chen YG. TGF- $\beta$ Signaling from Receptors to Smads. Cold Spring Harb Perspect Biol. 2016; 8(9): a022061.

97. Miyazono K, ten Dijke P, Heldin CH. TGF-beta signaling by Smad proteins. Adv Immunol. 2000; 75: 115-57.

98. Heldin $\mathrm{CH}$, Moustakas A. Role of Smads in TGF $\beta$ signaling. Cell Tissue Res. 2012; 347(1): 21-36

99. Kim YW, Park J, Lee HJ, et al. TGF- $\beta$ sensitivity is determined by N-linked glycosylation of the type II TGF- $\beta$ receptor. Biochem J. 2012; 45(3): 403-11.

100. Lin H, Wang D, Wu T, et al. Blocking core fucosylation of TGF- $\beta 1$ receptors downregulates their functions and attenuates the epithelial-mesenchymal transition of renal tubular cells. Am J Physiol Renal Physiol. 2011; 300(4): 1017-25

101. Kim YW, Park J, Lee HJ, et al. TGF- $\beta$ sensitivity is determined by N-linked glycosylation of the type II TGF- $\beta$ receptor. Biochem J. 2012; 445(3): 403-11.

102. Li LK, Wang N, Wang WD, et al. Blocking Posttranslational Core Fucosylation Ameliorates Rat Peritoneal Mesothelial Cell Epithelial-Mesenchymal Transition. Chin Med J (Engl). 2017; 130(18): 2147-55.

103. Lin $\mathrm{H}$, Wang $\mathrm{D}, \mathrm{Wu}$, et al. Blocking core fucosylation of TGF- $\beta 1$ receptors downregulates their functions and attenuates the epithelial-mesenchymal transition of renal tubular cells. Am J Physiol Renal Physiol. 2011; 300(4): 1017-25

104. Tu CF, Wu MY, Lin YC, et al. FUT8 promotes breast cancer cell invasiveness by remodeling TGF- $\beta$ receptor core fucosylation. Breast Cancer Res. 2017; 19(1): 111

105. Venkatachalam MA, Weinberg JM. New wrinkles in old receptors: core fucosylation is yet another target to inhibit TGF- $\beta$ signaling. Kidney Int. 2013; 84(1): 11-4.

106. Pinho SS, Seruca R, Gärtner F, et al. Modulation of E-cadherin function and dysfunction by N-glycosylation. Cell Mol Life Sci. 2011; 68(6): 1011-20.

107. Venhuizen JH, Jacobs FJC, Span PN, et al. P120 and E-cadherin: Double-edged swords in tumor metastasis. Semin Cancer Biol. 2020; 60: 107-20.

108. Zhu W, Leber B, Andrews DW. Cytoplasmic O-glycosylation prevents cell surface transport of E-cadherin during apoptosis. EMBO J. 2001; 20(21): 5999-6007.

109. Liwosz A, Lei T, Kukuruzinska MA. N-glycosylation affects the molecular organization and stability of E-cadherin junctions. J Biol Chem. 2006; 281(32): 23138-49.
110. Zhou F, Su J, Fu L et al. Unglycosylation at Asn-633 made extracellular domain of E-cadherin folded incorrectly and arrested in endoplasmic reticulum, then sequentially degraded by ERAD. Glycoconj J. 2008; 25(8): $727-40$.

111. Vagin O, Tokhtaeva E, Yakubov I, et al. Inverse correlation between the extent of N-glycan branching and intercellular adhesion in epithelia. Contribution of the Na,K-ATPase beta1 subunit. J Biol Chem. 2008; 283(4): 2192-202.

112. Liu D, Gao Z, Yue L. Fucosyltransferase 8 deficiency suppresses breast cancer cell migration by interference of the FAK/integrin pathway. Cancer Biomark. 2019; 25(4): 303-11.

113. Yang HF, Yu M, Jin HD, et al. Fentanyl Promotes Breast Cancer Cell Stemness and Epithelial-Mesenchymal Transition by Upregulating a1, 6-Fucosylation via Wnt/ $\beta$-Catenin Signaling Pathway. Front Physiol. 2017; 8: 510.

114. Hu P, Shi B, Geng F, et al. E-cadherin core fucosylation regulates nuclear beta-catenin accumulation in lung cancer cells. Glycoconj J. 2008; 25(9): 843-50.

115. Shao K, Chen ZY, Gautam S, et al. Posttranslational modification of E-cadherin by core fucosylation regulates Src activation and induces epithelial-mesenchymal transition-like process in lung cancer cells. Glycobiology. 2016; 26(2): 142-54.

116. Byrne AM, Sharma R, Duggan G, et al. Deoxycholic acid impairs glycosylation and fucosylation processes in esophageal epithelial cells. Glycobiology. 2012; 22(5): 638-48.

117. Goodman A, Patel SP, Kurzrock R. PD-1-PD-L1 immune-checkpoint blockade in B-cell lymphomas. Nat Rev Clin Oncol. 2017; 14(4): 203-20.

118. Ohaegbulam KC, Assal A, Lazar-Molnar E, et al. Human cancer immunotherapy with antibodies to the PD-1 and PD-L1 pathway. Trends Mol Med. 2015; 21(1): 24-33.

119. Okada M, Chikuma S, Kondo T, et al. Blockage of Core Fucosylation Reduces Cell-Surface Expression of PD-1 and Promotes Anti-tumor Immune Responses of T Cells. Cell Rep. 2017; 20(5): 1017-28.

120. Li CW, Lim SO, Xia W, et al. Glycosylation and stabilization of programmed death ligand-1 suppresses T-cell activity. Nat Commun. 2016; 7: 12632.

121. Sun L, Li CW, Chung EM, et al. Targeting Glycosylated PD-1 Induces Potent Antitumor Immunity. Cancer Res. 2020; 80(11): 2298-310.

122. Li CW, Lim SO, Xia W, et al. Glycosylation and stabilization of programmed death ligand-1 suppresses T-cell activity. Nat Commun. 2016; 7: 12632.

123. Okada M, Chikuma S, Kondo T, et al. Blockage of Core Fucosylation Reduces Cell-Surface Expression of PD-1 and Promotes Anti-tumor Immune Responses of T Cells. Cell Rep. 2017; 20(5): 1017-28.

124. Zhang N, Li M, Xu X, et al. Loss of core fucosylation enhances the anticancer activity of cytotoxic T lymphocytes by increasing PD-1 degradation. Eur J Immunol. 2020; 50(11): 1820-33.

125. Hynes RO. Integrins: bidirectional, allosteric signaling machines. Cell. 2002; 110: 673-87.

126. Thomas GJ, Nyström ML, Marshall JF. Alphavbeta6 integrin in wound healing and cancer of the oral cavity. J Oral Pathol Med. 2006; 35(1): 1-10.

127. Li ZH, Zhou Y, Ding YX, et al. Roles of integrin in tumor development and the target inhibitors. Chin J Nat Med. 2019; 17(4): 241-51.

128. Marsico G, Russo L, Quondamatteo F, et al. Glycosylation and Integrin Regulation in Cancer. Trends Cancer. 2018; 4(8): 537-52.

129. Gu J, Taniguchi N. Regulation of integrin functions by N-glycans. Glycoconj J. 2004; 21(1-2): 9-15.

130. Janik ME, Lityńska A, Vereecken P. Cell migration-the role of integrin glycosylation. Biochim Biophys Acta. 2010; 1800(6): 545-55.

131. Zhao Y, Itoh S, Wang X, et al. Deletion of core fucosylation on alpha3beta1 integrin down-regulates its functions. J Biol Chem. 2006; 281(50): 38343-50.

132. Kalaydina RV, Zhou H, Markvicheva E, et al. Impact of Fucosylation on Self-Assembly of Prostate and Breast Tumor Spheroids by Using Cyclo-RGDfK(TPP) Peptide and Image Object Detection. Onco Targets Ther. 2019; 12: 11153-73.

133. Elvin JG, Couston RG, van der Walle CF. Therapeutic antibodies: market considerations, disease targets and bioprocessing. Int J Pharm. 2013; 440(1): 83-98.

134. Iannello A, Ahmad A. Role of antibody-dependent cell-mediated cytotoxicity in the efficacy of therapeutic anti-cancer monoclonal antibodies. Cancer Metastasis Rev. 2005; 24(4): 487-99.

135. Talathi SP, Shaikh NN, Pandey SS, et al. FcyRIIIa receptor polymorphism influences NK cell mediated ADCC activity against HIV. BMC Infect Dis. 2019; 19(1): 1053.

136. Jefferis, R. Glycosylation as a strategy to improve antibody-based therapeutics. Nat Rev Drug Discov. 2009, 8: 226-34.

137. Malphettes L, Freyvert Y, Chang J, et al. Highly efficient deletion of FUT8 in $\mathrm{CHO}$ cell lines using zinc-finger nucleases yields cells that produce completely nonfucosylated antibodies. Biotechnol Bioeng. 2010; 106(5): 774-83.

138. Shields RL, Lai J, Keck R, et al. Lack of fucose on human IgG1 N-linked oligosaccharide improves binding to human Fcgamma RIII and antibody-dependent cellular toxicity. J Biol Chem. 2002; 277(30): 26733-40.

139. Beck A, Reichert JM. Marketing approval of mogamulizumab: a triumph for glyco-engineering. MAbs. 2012; 4(4): 419-25.

140. Pereira NA, Chan KF, Lin PC, et al. The "less-is-more" in therapeutic antibodies: Afucosylated anti-cancer antibodies with enhanced antibody-dependent cellular cytotoxicity. MAbs. 2018; 10(5): 693-711.

141. Cartron G, de Guibert S, Dilhuydy MS, et al. Obinutuzumab (GA101) in relapsed/refractory chronic lymphocytic leukemia: final data from the phase 1/2 GAUGUIN study. Blood. 2014; 124(14): 2196-202. 
142. Salles GA, Morschhauser F, Solal-Céligny P, et al. Obinutuzumab (GA101) in patients with relapsed/refractory indolent non-Hodgkin lymphoma: results from the phase II GAUGUIN study. J Clin Oncol. 2013; 31(23): 2920-26.

143. Goede V, Fischer K, Busch R, et al. Obinutuzumab plus chlorambucil in patients with CLL and coexisting conditions. N Engl J Med. 2014; 370(12): 1101-10.

144. Omasa T, Onitsuka M, Kim WD. Cell engineering and cultivation of chinese hamster ovary (CHO) cells. Curr Pharm Biotechnol. 2010; 11(3): 233-40.

145. Malphettes L, Freyvert Y, Chang J, et al. Highly efficient deletion of FUT8 in $\mathrm{CHO}$ cell lines using zinc-finger nucleases yields cells that produce completely nonfucosylated antibodies. Biotechnol Bioeng. 2010; 106(5): 774-83.

146. Yamane-Ohnuki N, Kinoshita S, Inoue-Urakubo M, et al. Establishment of FUT8 knockout Chinese hamster ovary cells: an ideal host cell line for producing completely defucosylated antibodies with enhanced antibody-dependent cellular cytotoxicity. Biotechnol Bioeng. 2004; 87(5): 614-22.

147. Zong H, Han L, Ding K, et al. Producing defucosylated antibodies with enhanced in vitro antibody-dependent cellular cytotoxicity via FUT8 knockout CHO-S cells. Eng Life Sci. 2017; 17(7): 801-8.

148. Lan Y, Hao C, Zeng X, et al. Serum glycoprotein-derived N- and O-linked glycans as cancer biomarkers. Am J Cancer Res. 2016; 6(11): 2390-415.

149. Liao C, An J, Tan Z, et al. Changes in Protein Glycosylation in Head and Neck Squamous Cell Carcinoma. J Cancer. 2021; 12(5): 1455-66.

150. Liu YC, Yen HY, Chen CY, et al. Sialylation and fucosylation of epidermal growth factor receptor suppress its dimerization and activation in lung cancer cells. Proc Natl Acad Sci U S A. 2011; 108(28): 11332-7.

151. Wang $\mathrm{X}, \mathrm{Gu} \mathrm{J}$, Ihara $\mathrm{H}$, et al. Core fucosylation regulates epidermal growth factor receptor-mediated intracellular signaling. J Biol Chem. 2006; 281: 2572-7. 